\title{
Capacitorless DC-DC Regulator as a Candidate Topology for Photovoltaic Solar Facilities
}

\author{
Herminio Martínez-García \\ Barcelona College of Industrial Engineering (EUETIB) \\ Department of Electronics Engineering \\ Technical University of Catalonia (UPC). BarcelonaTech \\ Campus de la Escuela Industrial de Barcelona. C/ Comte d'Urgell, $\mathrm{n}^{\circ} 187$ \\ 08036 - Barcelona. SPAIN \\ Phone Number: +34.93.413.72.90; Fax Number: +34.93.413.74.01; E-mail: $\underline{\text { herminio.martinez@upc.edu }}$
}

\begin{abstract}
Linear-assisted DC/DC converters (or linearswitching hybrid DC/DC converters) consist of a voltage linear regulator (classic NPN or nMOS topologies and LDO) connected in parallel with a switching DC/DC converter. They are a good candidate for energy processing in photovoltaic solar facilities. In order to control these hybrid structures, different strategies exist, allowing fixing the switching frequency as a function of some parameters of the linear regulator. This article compares two control strategies that, although can be applied to the same circuital structure of linear-assisted converter, are sensibly different. The first one, reported in previous literature, cancels completely the average current through the linear regulator in steady state to achieve a reduction of the losses. Thus the efficiency of the whole system increases and almost equals the one of the standalone switching converter. The proposed approach, in spite of a slightly increment of linear regulator's losses, reduces the output ripple due to the crossover distortion of linear regulator output stage.
\end{abstract}

\section{Key words}

DC-DC Switching Converters, Voltage Linear Regulators, Linear-Assisted DC-DC Voltage Regulators.

\section{Introduction}

Two different alternatives have been widely used for decades to provide the necessary power supply voltage to electronics circuits and systems. These two alternatives are known largely: (1) the use of voltage series linear regulators (classic standard NPN - or nMOS- topologies and LDO) [1]-[3], and (2) DC/DC switching converters, thanks to which high current and high efficiency power supply systems can be obtained [4]-[6].

However, a third alternative, linear-assisted DC/DC converters (also known as linear-switching hybrid converters) is possible. They are circuital structures that present an increasing interest for the implementation of power supply systems that require two demanding design specifications: (1) high slew-rate of the output current and
(2) high current consumption by the output load. This it is the case of the systems based on the modern microprocessors and DSPs, where both requirements converge [7], [8]. These linear-switching hybrid converters are able to combine the well-known advantages of the two existing typical alternatives for the implementation of $\mathrm{DC} / \mathrm{DC}$ voltage regulators or converters, diminishing as well their disadvantages. Linear-assisted DC/DC converters can be implemented on printed circuits using discrete components. Nevertheless, they are also an attractive alternative susceptible to be integrated in on-chip power supply systems as a part of power management systems.

The basic scheme of a linear-assisted converter is shown in figure 1 [9], [10]. This structure consists, mainly, of a voltage linear regulator in parallel with a step-down switching DC/DC converter. In this type of converters, the value of the output voltage, supposed constant, is fixed with good precision by the voltage linear regulator. The current through this linear regulator is constantly sensed by the current sense element Rm. Based on its value, the controller activates or not the output of comparator CMP1 that controls the switching element of the DC/DC converter. Notice that the current through the linear regulator constitutes a measurement of the error of the power supply system.

The power stage (that is, the switching converter) injects at the output the current required to force to a minimum value (not necessarily zero) the current through the linear regulator. As a consequence, it is obtained, altogether, a power supply system where the switching frequency comes fixed, among other parameters (such as the possible hysteresis of the analog comparator), by the value of the current through the linear regulator.

As an additional advantage of the structure shown in figure 1, and in contrast to which happens in other hybrid structures that work in open loop [11], it is possible to 
emphasize that the use of filters in the respective outputs of the linear and switching blocks (and, therefore, the possible optimization or equalization of its group propagation delays) is not, in this case, necessary. The present article allows to compare two strategies of control that can be applied to the same circuital structure of a linear-assisted converter. However, they are significantly different. The first one (that we will denominate ' $A$ ' and is reported in previous literature [12],[13]), tries to cancel completely the current through the linear regulator in the steady state in order to achieve a reduction of the losses. Thus the efficiency of the whole system increases and almost equals the one of the switching converter. It considers as a "main" block the switching DC/DC converter, and the linear regulator as an auxiliary module.

On the other hand, this proposal (strategy ' $B$ ') allows some average current flowing through the linear regulator. In spite of a slightly increasing of linear regulator's losses, this strategy reduces the output ripple due to the crossover distortion of its output stage. Thus, this approach considers as a "main" block the linear one, and the switching one as an auxiliary module.

In Section 2, the strategy of control $A$ is discussed and shows its advantages and drawbacks. In Section 3 the strategy of control $B$ is compared with the first one. In Section 4 the linear-assisted converter is modified to improve its transient response. The article concludes with the main conclusions in Section 5.

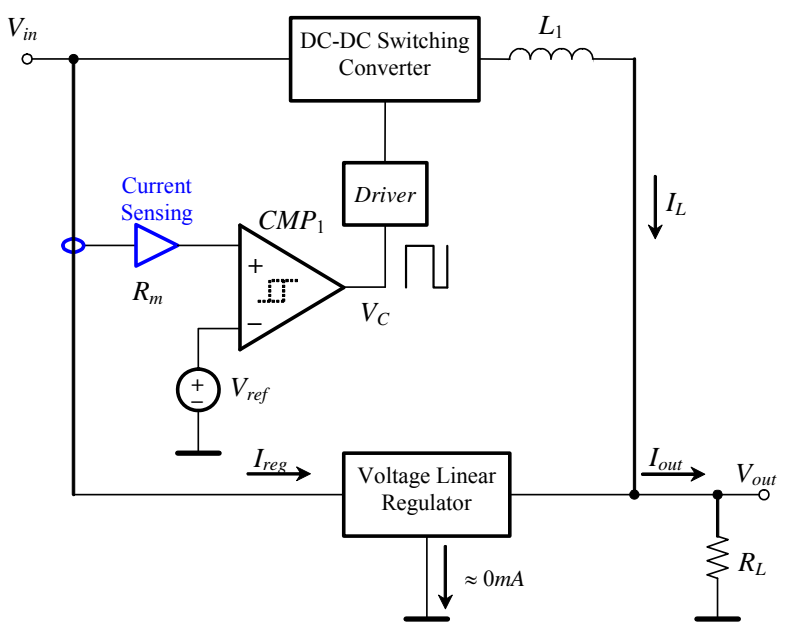

Fig. 1. Block diagram of a linear-assisted converter.

\section{Control Strategy ' $A$ '}

The first of the two strategies of control that is considered in the current article is implemented on the converter of figure 2, where the implementation of the linear-assisted converter consists of a linear regulator (including transistors $Q_{2 a}$ and $Q_{2 b}$, which form an output complementary push-pull stage) and a switching DC/DC converter connected in parallel with the first one. In this case, the switching converter is a step-down type (buck converter) without the output capacitance. With this strategy, the switching converter is considered as a "main" block, whereas the linear regulator is considered as the auxiliary block that "assists" the first one when it is not able to provide output currents with high variations (that is to say, with high slew rate of the load current).

The control strategy consists of sensing the current through the linear regulator and, transforming it into a voltage (thanks to the current sensing element $\mathrm{Rm}$ ), controlling the switching frequency of the DC/DC switching converter. The main objective of this one is to provide all the load current in steady-state conditions (to obtain high efficiency of the whole system). Thus, in steady state the linear regulator does not provide current to the load, although it maintains the output voltage to an acceptable DC value. However, when variable output loads are driven, the linear regulator provides high transitory changes of the current in order to maintain constant the output voltage of the whole structure (figure 3). Therefore, we can name to this type of control as strategy control with null average linear regulator current. Resistors $R_{1}$ and $R_{2}$ of the Schmitt trigger determine the width of its hysteresis cycle and, thus, the maximum value of the switching frequency of the $\mathrm{DC} / \mathrm{DC}$ converter.

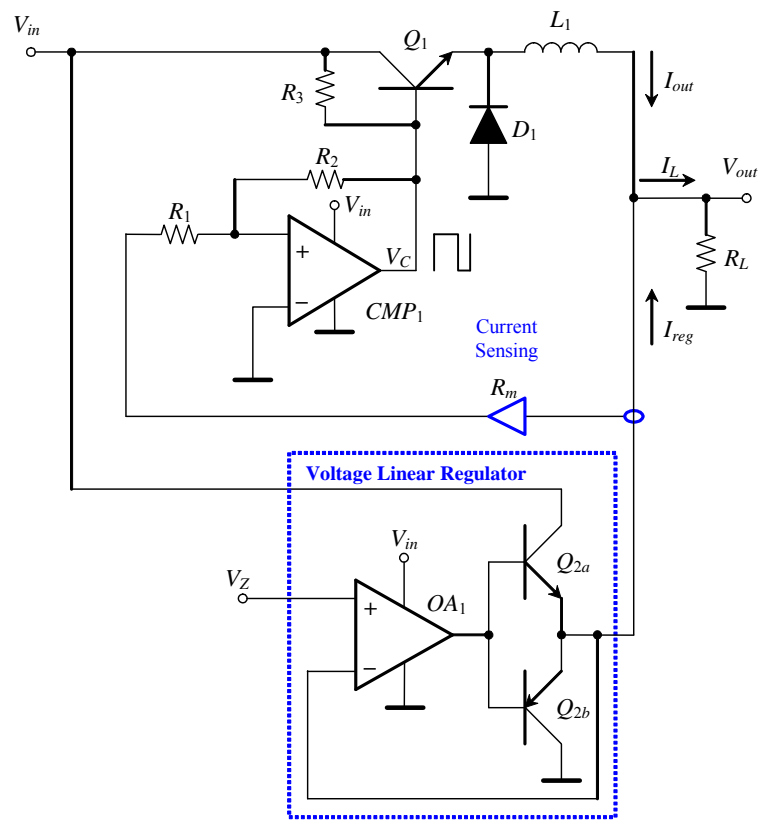

Fig. 2. Basic structure of a linear-assisted converter to implement the control strategy ' $A$ '.

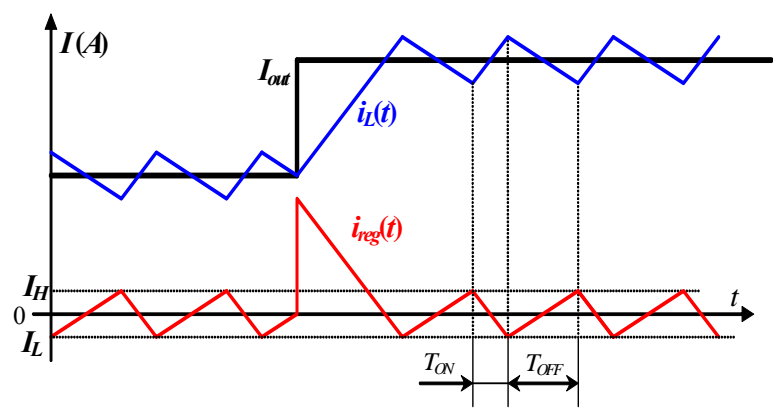

Fig. 3. Principle of operation of a linear-assisted converter with control strategy ' $A$ '. 


\section{Control Strategy ' $B$ '}

The proposed strategy is analyzed using the step-down switching converter shown in figure 4 [14], [15]. The linear regulator consists of a push-pull output stage (transistors $Q_{2 a}$ and $Q_{2 b}$ ). In this strategy, the main objective of the DC-DC switching converter is to provide most of the load current in steady-state conditions to obtain also a good efficiency of the whole system. Thus, thanks to the incorporation of the reference voltage $V_{\text {ref }}$ at the inverting input of the analog comparator, the linear regulator provides a small part of the load current in steady state, maintaining the output voltage to an acceptable constant value.

As a matter of fact, if the current demanded by the load is inferior to a maximum value of current, which we will denominate switching threshold current, $I_{\gamma}$, the output of comparator $C M P_{1}$ will be at low level, disabling the DC/DC switching converter and, thus, the current through inductor $L_{1}$ will be zero. Therefore, the voltage linear regulator supplies the load $R_{L}$, providing all the output current $\left(I_{\text {reg }}=I_{\text {out }}\right)$.

When the current demanded by the load overpasses this current limit $I_{\gamma}$, automatically the output of the comparator will pass to high level, causing that the current through the inductance $L_{1}$ grows linearly according to:

$$
i_{L}(t)=\frac{V_{\text {in }}-V_{\text {out }}}{L_{1}} t+I_{L}\left(\tau_{1}\right)
$$

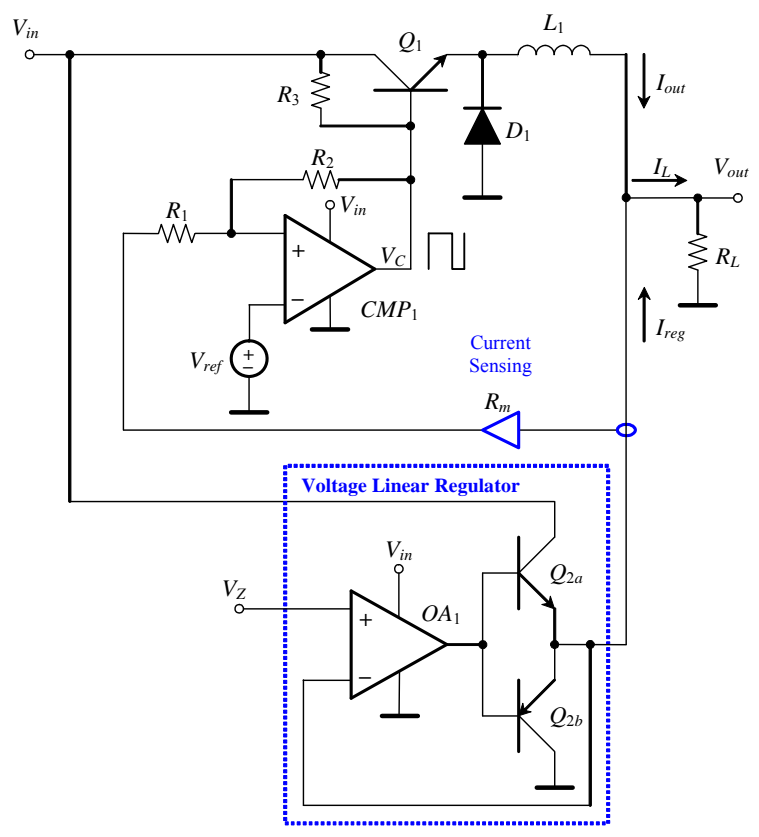

Fig. 4. Basic structure of linear-assisted converter with strategy of control ' $B$ '.

In that expression, the conduction collector-emitter voltage of transistor $Q_{1}$ is ignored. $I_{L}\left(\tau_{1}\right)$ is the initial value of the current through inductor $L_{1}$ at the time instant $T_{O N}$. Considering that the output current $I_{\text {out }}=I_{\text {reg }}+I_{L}$, and is assumed to be constant (equal to $V_{\text {out }} / R_{L}$ ), the linear regulator current $I_{\text {reg }}$ will decrease linearly, until becoming slightly smaller than $I_{\gamma}$. At this moment, the comparator will change its output to low level, cutting the transistor $Q_{1}$ and causing that the current through the inductor decreases according to equation (2):

$$
i_{L}(t)=-\frac{V_{\text {out }}}{L_{1}} t+I_{L}\left(\tau_{2}\right)
$$

In this expression it is considered that the diode $D_{1}$ is ideal (with zero direct voltage). $I_{L}\left(\tau_{2}\right)$ is the maximum value reached by the current flowing through the inductor (just at the beginning of the interval $T_{\text {OFF }}$ ). When the inductor current decreases to a value in which $I_{r e g}>I_{\gamma}$, the comparator changes its state to high level, repeating the cycle again.

Without hysteresis in the comparator, the switching point of the $\mathrm{DC} / \mathrm{DC}$ switching converter is given by the switching threshold current, $I_{\gamma}$, of the linear regulator. This one can be adjusted to a value thanks to the gain of the current sensing element, $R_{m}$, and the reference voltage $V_{\text {ref, }}$ according to the expression:

$$
I_{\gamma}=\frac{V_{r e f}}{R_{m}}
$$

In case of a comparator without hysteresis, intrinsic delays of the electronic circuits determine a small hysteresis that limits the maximum value of the linearassisted converter switching frequency (figure 5). However, with the objective of fixing this switching frequency to a practical value, in order not to increase significantly losses by the switching process, it is important to add the aforementioned hysteresis to the comparator $C M P_{1}$.

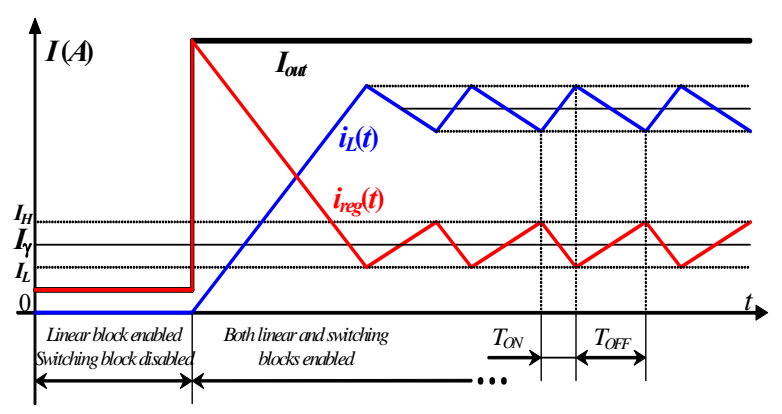

Fig. 5. Principle of operation of a linear-assisted converter with strategy of control ' $B$ '.

Notice that if the load current is below $I_{\gamma}$, the switching block (the DC/DC converter) is disabled in order to minimize its losses. Thus, only the linear regulator provides the output current for slight load conditions. Figure 6 shows the experimental efficiency versus the load current for the two strategies. It is shown the comparison of the control strategy ' $A$ ' and the proposed by authors (strategy ' $B$ ') for $V_{\text {out }}=5 \mathrm{~V}$. It is shown four different $I_{\gamma}$ values: $0 \mathrm{~mA}$ (strategy ' $A$ '), and $10 \mathrm{~mA}, 50$ $m A$ and $100 m A$ (all three for strategy ' $B$ '). Note that, if $I_{\gamma}$ 
is low, the efficiency is not almost affected, reducing the output ripple too.

\section{Improvement of the Linear-Assisted Converter with Control Strategy ' $B$ '}

Studying the preceding figures, it is possible to ask for the necessity or not of using the transistor PNP $Q_{2 b}$ in the scheme of figure 7 , since this one, in steady state (and unlike the strategy $A$ ), always remains in cut. However, the inclusion of the same one is necessary to increase the response speed of the linear regulator from decremental variations of the load current. In fact, since it has been reflected in the preceding figures, one of the objectives of the linear regulator, besides obtaining an excellent regulation of the output voltage, frees of ripples, is to provide fast current responses when abrupt variations of the load consumption exist. In this way, the switching DC/DC converter cannot respond to those variations of the output current. Thus, this current transients must be provided (or absorbed, depending on if the variation of the load current is incremental or decremental) by the linear regulator.

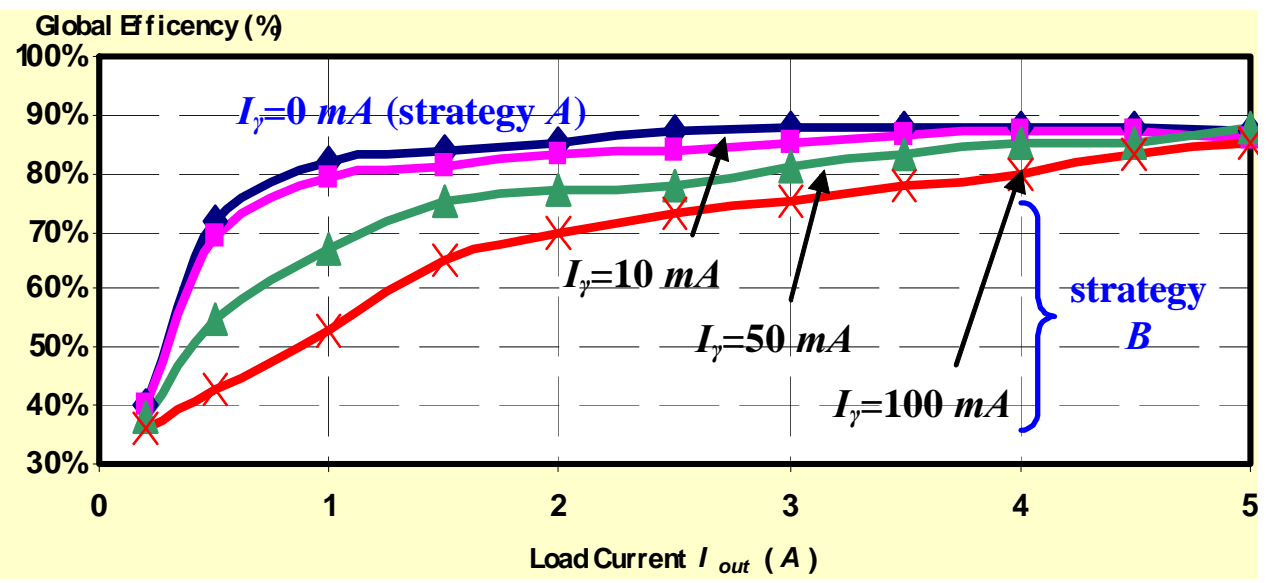

Fig. 6. Experimental efficiency for the control strategy $A$ and the proposed by the authors (strategy $B$ ) with $V_{\text {out }}=5 \mathrm{~V}$. It is shown four different $I_{\gamma}$ values: $0 \mathrm{~mA}$ (strategy $A$ ), and $10 \mathrm{~mA}, 50 \mathrm{~mA}$ and $100 \mathrm{~mA}$ (all three for strategy $B$ ).

In the previous figures this can be appreciated when the consumption of the load increases from an initial value (in this case $1 A$ ) to another end value greater (equal to $2 A$ ). Nevertheless, it is important to notice that, if the response wants also to be maintained for descendent variations of the load current, the voltage linear regulator must incorporate necessarily the transistor $Q_{2 b}$ (in both figure 2 and figure 4) to allow bidirectional output currents. we have an increasing step of the load current at $t=40 \mu \mathrm{s}$ and a decreasing variation at $t=60 \mu \mathrm{s}$. Note that the answer provided by the linear regulator for ascending variations of the load current is good enough, and allows, therefore, to maintain the output voltage of the set constant during the transient. However, the response of the set is not appropriate for descendent variations, being the response time of the set marked by the switching converter, losing the load regulation at that time interval.

In figure 7 we can appreciate the response of the proposed hybrid convert in figure 4 without the $Q_{2 b}$ transistor, when

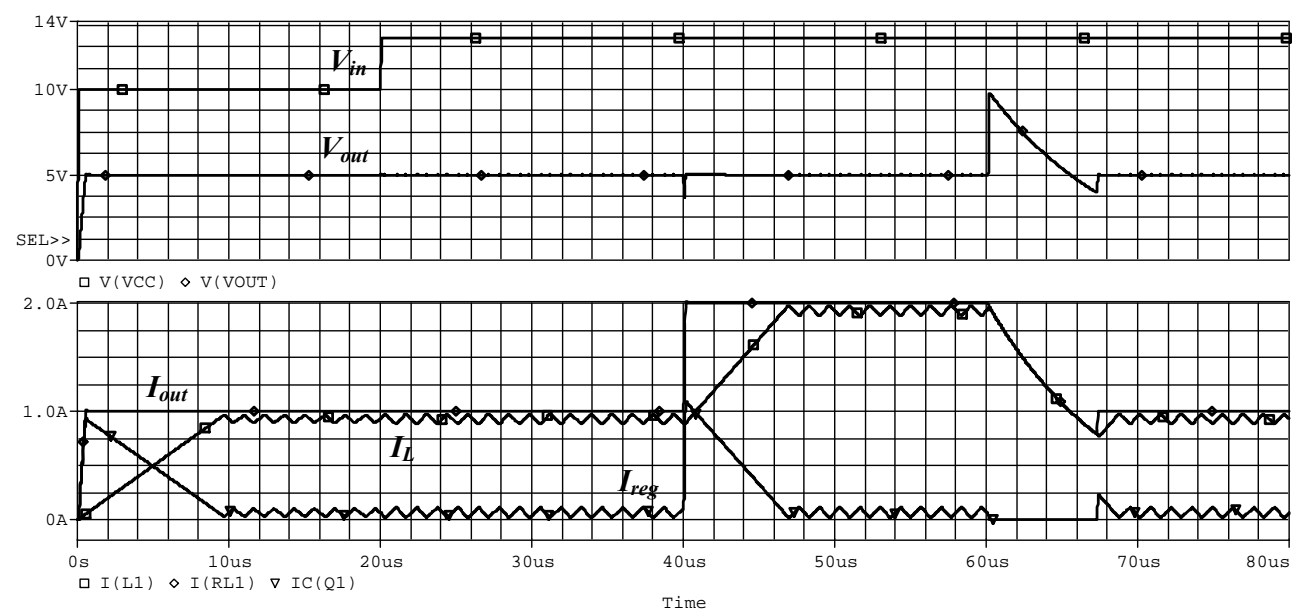

Fig. 7. Detail of the lack of load regulation when the load current changes from $2 A$ to $1 A$ at $t=60 \mu$ s for the converter in figure 4 without the transistor $Q_{2 b}$ (variation in the load resistance from $2.5 \Omega$ to $5 \Omega$ ). The switching threshold current is adjusted to $50 \mathrm{~mA}$. 
However, if in the hybrid converter in figure 4 transistor PNP $Q_{2 b}$ is added, the response of the converter improves significantly when the load current decreases. In figure 8 we can appreciate that, when a decreasing step of the output current takes place from $2 A$ to a $1 A$ at $t=60 \mu \mathrm{s}$, the transistor $Q_{2 b}$ included in the linear regulator can absorb the excess of current that, provided by the inductor $L_{1}$, the load no longer accepts. Thus, the linear regulator can maintain an output voltage with good regulation (and, therefore, free of ripples) even with decreasing transients. Finally, figure 9 shows experimental details of the load regulation when the load current changes from $5 \mathrm{~A}$ to 1.4 $A$ for the implemented converter in figure 4 without (figure 9.a) and with (figure 9.b) the transistor $Q_{2 b}$. The switching threshold current is adjusted to $50 \mathrm{~mA}$.
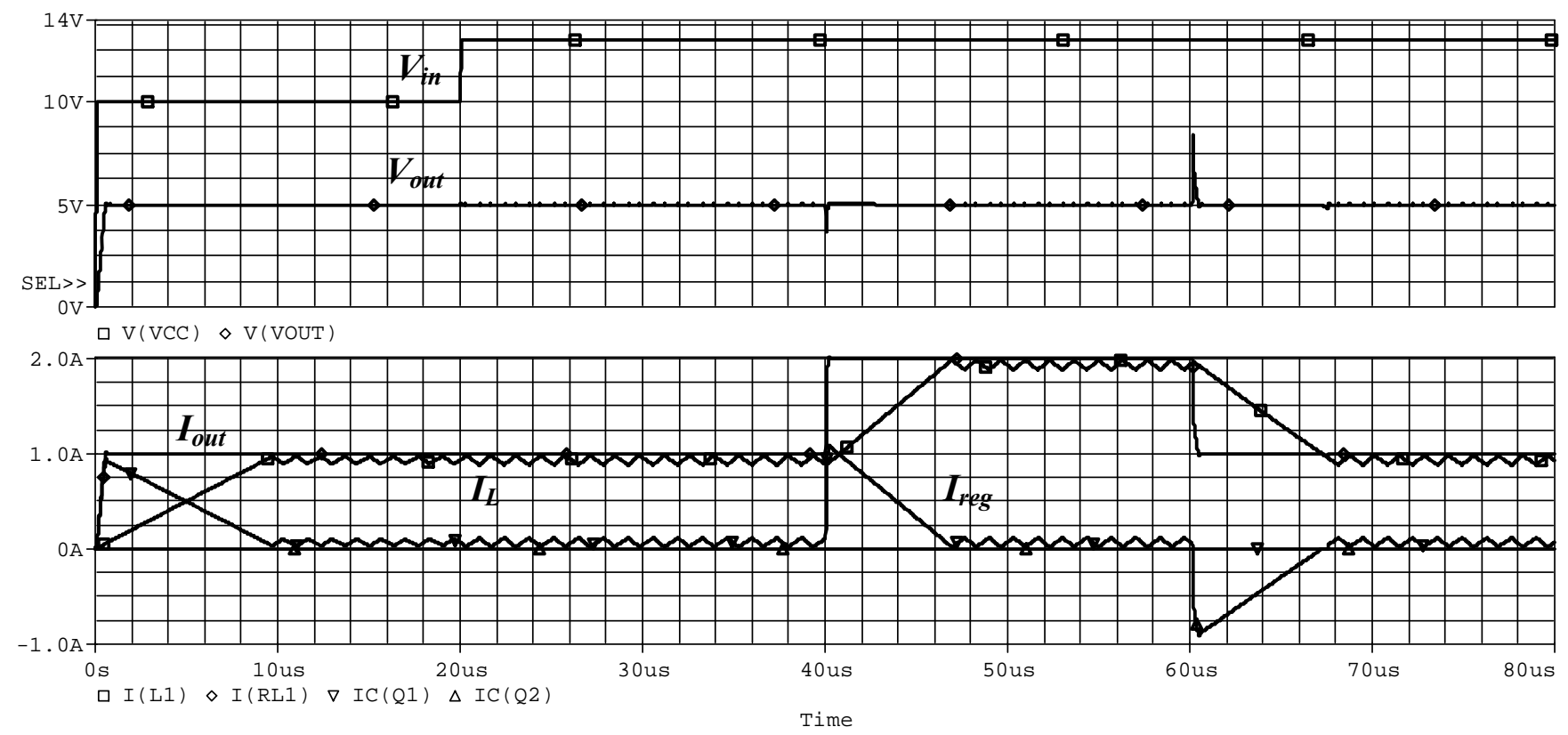

Fig. 8. Detail of the improvement obtained in the load regulation when the load current changes from $2 A$ to $1 A$ at the time instant $t=60 \mu$ s for the converter in figure 4 with the transistor $Q_{2 b}$ (variation in the load resistance from $2.5 \Omega$ to $5 \Omega$ ). The switching threshold current is adjusted to $50 \mathrm{~mA}$.

\section{Conclusion}

The present article has shown the comparative of two strategies of control sensibly different for power DC/DC linear-assisted (or hybrid) converters based on the association of a linear regulator in parallel with a switching converter. The first of the two strategies (strategy ' $A$ ' or with null average value in the linear regulator current) allows to obtain a high efficiency, similar to switching converters because, in the steady state, the power dissipated in the linear regulator is practically zero. However, it has as inconvenient the presence of a ripple output voltage because the pass transistor of the output linear stage is switching between the cut and the conduction in every switching period.

The proposal presented in the article (strategy of control ' $\mathrm{B}$ ' or nonnull average value in the linear regulator current), allows a little current through the linear stage that causes that the efficiency of the set diminishes slightly. However, it allows to obtain an output voltage practically free of spurious ripples.

\section{Acknowledgement}

This work has been partially supported by the Spanish Ministerio de Economía y Competitividad by project DPI2013-47799-C2-2-R.

\section{References}

[1] C. K. Chava, J. Silva-Martínez. "A Frequency Compensation Scheme for LDO Voltage Regulators". IEEE Transactions on Circuits and Systems-I: Regular Papers, vol. 51 ( $\mathrm{n}^{\circ}$ 6): pp. 1041-1050, June 2004.

[2] R. J. Milliken, J. Silva-Martínez, E. Sánchez-Sinencio. "Full On-Chip CMOS Low-Dropout Voltage Regulator". IEEE Transactions on Circuits and Systems-I: Regular Papers, vol. 54 ( ${ }^{\circ}$ 9): pp. 1879-1890, September 2007.

[3] V. Grupta, G. A. Rincón-Mora, P. Raha. 'Analysis and Design of Monolithic, High PSR, Linear Regulator for SoC Applications'. Proceedings of the IEEE International SoC Conference: pp. 311-315, 2004.

[4] R. W. Erickson, D. Maksimovic. 'Fundamentals of Power Electronics'. $2^{\text {nd }}$ edition, Ed. Kluwer Academic Publishers, 2001.

[5] J. G. Kassakian, M. F. Schlecht, G. C. Verghese. 'Principles of Power Electronics'. Ed. Addison-Wesley, 1991.

[6] N. Mohan, T. M. Underland, W. P. Robbins. 'Power Electronics: Converters, Applications and Design'. Ed. John Wiley \& Sons, 1989.

[7] X. Zhou, P. L. Wong, P. Xu, F. C. Lee, A. Q. Huang. 
'Investigation of Candidate VRM Topologies for Future Microprocessors'. IEEE Transactions on Power Electronics, vol. 15 ( $\mathrm{n}^{\circ}$ 6): pp. 1172-1182, November 2000.

[8] B. Arbetter, D. Maksimovic. 'DC-DC Converter with Fast Transient Response and High Efficiency for LowVoltage Microprocessor Loads'. IEEE Applied Power Electronics Conference, pp. 156-162. 1998.

[9] P. Midya, F. H. Schlereth. 'Dual Switched Mode Power Converter'. IECON. Industrial Electronics Society: pp. $155-158,1989$.

[10] F. H. Schlereth, P. Midya. 'Modified Switched Power Convertor with Zero Ripple'. Proceedings of the $32^{\text {nd }}$ IEEE Midwest Symposium on Circuits and Systems (MWSCAS’90): pp. 517-520, 1990.

[11] V. Yousefzadeh, E. Alarcón, D. Maksimovic. 'Band Separation and Efficiency Optimization in LinearAssisted Switching Power Amplifiers'. Proceedings of the 37th IEEE Power Electronics Specialists Conference, 2006 (PESC'06), pp. 1-7, 18-22 June 2006.

[12] R. Vázquez, A. Barrado, E. Olías, A. Lázaro. 'Theoretical Study and Implementation of a High Dynamic Performance, High Efficiency and Low Voltage Hybrid Power Supply'. Proceedings of the IEEE 32nd Annual

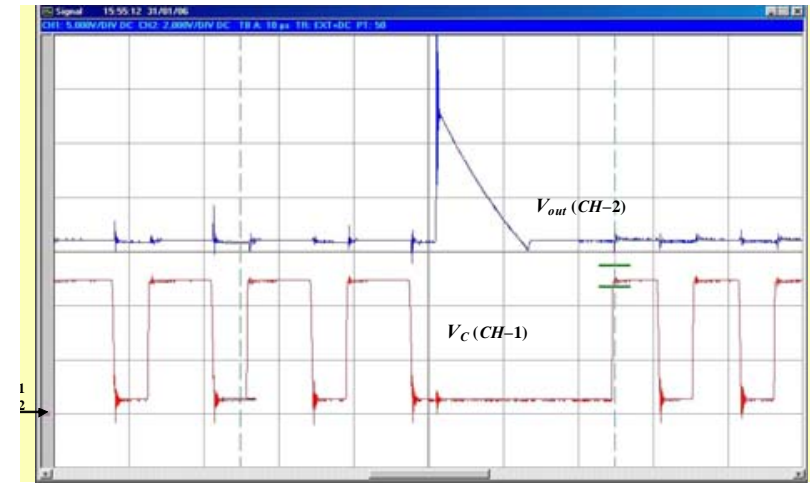

(a)
Power Electronics Specialists Conference, 2001 (PESC 2001), vol. 3: pp. 1517-1522. 17-21 June 2001.

[13] A. Barrado, R. Vázquez, E. Olías, A. Lázaro, J. Pleite. 'Theoretical Study and Implementation of a Fast Transient Response Hybrid Power Supply'. IEEE Transactions on Power Electronics, vol. 19 ( $\left.\mathrm{n}^{\mathrm{o}} 4\right)$ : $\mathrm{pp}$. 1003-1009, July 2004.

[14] H. Martínez, A. Conesa. "Modeling of Linear-Assisted DC-DC Converters". European Conference on Circuit Theory and Design 2007 (ECCTD 2007), 26th-30th August 2007.

[15] A. Conesa, H. Martínez, J. M. Huerta. "Modeling of Linear \& Switching Hybrid DC-DC Converters". 12th European Conference on Power Electronics and Applications (EPE 2007), September 2007.

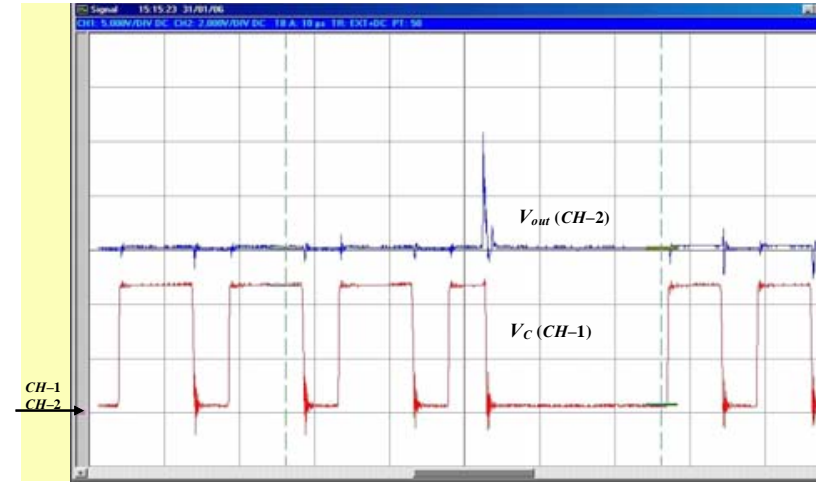

(b)

Fig. 9. Experimental results of the load regulation when the load current changes from $5 \mathrm{~A}$ to $1.4 \mathrm{~A}$ for the implemented converter in Fig. 4 without and with the transistor $Q_{2 b}$. The switching threshold current is adjusted to $50 \mathrm{~mA}$. 